\section{Co-infection with SARS-CoV-2 and Influenza A Virus in Patient with Pneumonia, China}

\author{
Xiaojing Wu, Ying Cai, Xu Huang, Xin Yu, Li Zhao, \\ Fan Wang, Quanguo Li, Sichao Gu, Teng Xu, \\ Yongjun Li, Binghuai Lu, Qingyuan Zhan
}

Author affiliations: China-Japan Friendship Hospital, Beijing, China (X. Wu, Y. Cai, X. Huang, X. Yu, L. Zhao, S. Gu, B. Lu, Q. Zhan); The Sixth Medical Center of PLA General Hospital, Beijing (F. Wang); Weifang No. 2 People's Hospital, Weifang, China (Q. Li); Vision Medicals Co., Ltd., Guangzhou, China (T. Zu, Y. Li)

\section{DOI: https://doi.org/10.3201/eid2606.200299}

We report co-infection with severe acute respiratory syndrome coronavirus 2 (SARS-CoV-2) and influenza A virus in a patient with pneumonia in China. The case highlights possible co-detection of known respiratory viruses. We noted low sensitivity of upper respiratory specimens for SARS-CoV-2, which could further complicate recognition of the full extent of disease.

Tn December 2019, a series of cases of pneumonia Lof unknown cause was reported in Wuhan, Hubei Province, China. On January 7, 2020, the causative pathogen was identified as a virus subsequently named severe acute respiratory syndrome coronavirus 2 (SARS-CoV-2) (1-3). We report a case of co-infection with SARS-CoV-2 and influenza A virus in China.

A 69-year-old man was seen in the clinic of China-Japan Friendship Hospital on January 23, 2020, for fever and dry cough. The patient visited Wuhan from December 18, 2019-January 22, 2020, and began having symptoms January 23. He reported no underlying medical conditions. Routine blood tests revealed a leukocyte count of $5.70 \times 10^{9}$ cells/L (reference range 3.5-9.5 $\times 10^{9}$ cells/L) and lymphocyte count of $2.18 \times 10^{9}$ cells/L (reference range 1.1-3.2 $\times$ $10^{9}$ cells/L). Chest computed tomography revealed a mass, ground-glass consolidation in the right inferior lobe of the lungs (Figure, panel A). Because of the patient's travel history, he was isolated for suspected coronavirus disease (COVID-19).

We obtained a nasopharyngeal swab specimen and conducted real-time reverse transcription-PCR (rRT-PCR) for SARS-CoV-2 by using reagents provided by Shanghai BioGerm Medical Technology Co., Ltd. (http://www.bio-germ.com), and Da An Gene Co., Ltd. (Sun Yat-Sen University, http:// en.daangene.com), on a LightCycler 480 (Roche, https://lifescience.roche.com). However, both tests returned negative results 8 hours later. We obtained another nasopharyngeal swab specimen for detection of SARS-CoV-2 and for differentiation of influenza $A$ and $B$ and respiratory syncytial viruses by using Xpert Flu/RSV Xpress assay (Cepheid, https:/ / www.cepheid.com). The sample was negative for SARS-CoV-2 but positive for influenza A. The patient was discharged with oral oseltamivir and instructed to stay home for isolation.

On January 30, the patient returned to the hospital reporting persistent fever and aggravated dyspnea. Routine blood tests showed a leukocyte count of $8.23 \times 10^{9}$ cells/L and lymphocyte count of $0.77 \times 10^{9}$ cells/L. A chest radiograph showed diffuse exudative shadows in bilateral lungs, indicating acute respiratory distress syndrome (Figure, panel B). Physical examination revealed respiratory rate of 30 breaths/min and oxygen saturation of $83 \%$ on ambient air. We administered oxygen and screened another nasopharyngeal swab specimen, which was negative for SARS-CoV-2. Considering his clinical features, we performed a fourth test for SARS-CoV-2 by using a sputum sample, which also was negative. The patient's dyspnea and
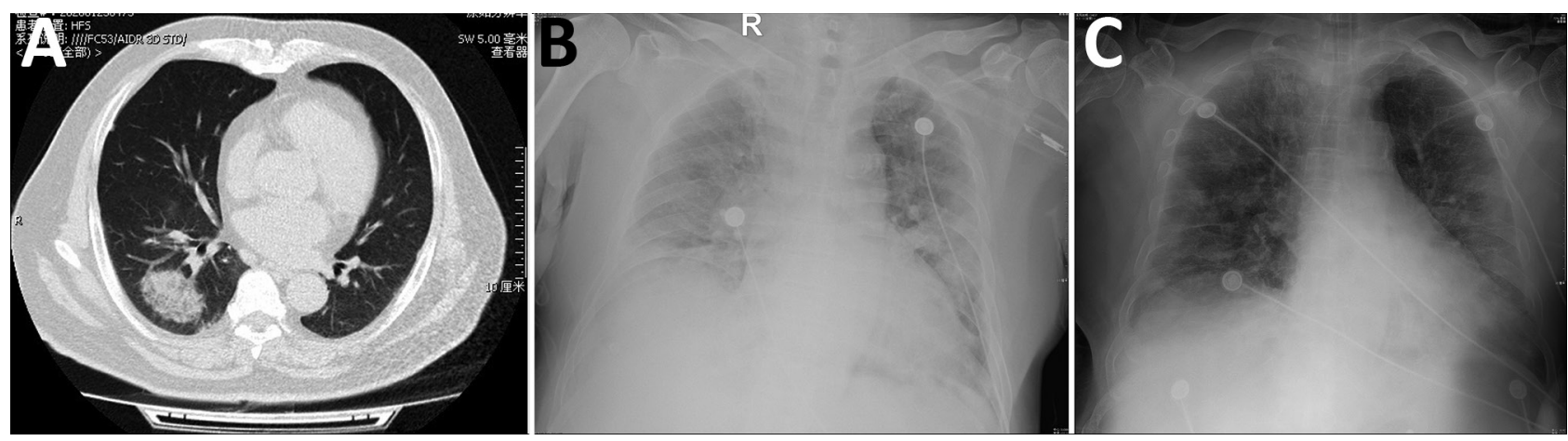

Figure. Radiographs of patient co-infected with severe acute respiratory syndrome coronavirus 2 and influenza A virus, China, 2020. A) Chest computed tomography demonstrating a mass, ground-glass consolidation in the right inferior lobe. B) Chest radiograph showing bilateral diffuse exudative shadows, indicating acute respiratory distress syndrome. C) Chest radiograph showing improved lung fields after 4 days in the intensive care unit. 
respiratory distress increased, and his oxygenation index was <200. We admitted the patient to the single negative-pressure ward of the medical intensive care unit for severe influenza A pneumonia and administered endotracheal intubation because of severe hypoxemia.

Four days later, the patient's oxygenation and chest radiographs improved (Figure, panel C). We performed a bronchoscopy and obtained bronchoalveolar lavage fluid (BALF) for metagenomic next-generation sequencing (mNGS) to identify potential pathogens. On February 5, mNGS reported 3,460 sequences that showed $99.8 \%$ identity and covered $98.69 \%$ of the SARS-CoV-2 genome NC_045512.2 |SARS-CoV-2|Wuhan-Hu-1 (GenBank accession no. NC_045512.2). We then performed rRTPCR by using newly collected sputum and stored BALF, which also tested positive. Cycle threshold values were 34 for sputum and 30 for BALF. However, a fourth nasopharyngeal swab collected concurrently with the second sputum sample remained negative. The next day, the patient was transferred to a designated hospital for further critical care.

This case highlights 2 challenges in the diagnosis of COVID-19. First, the sensitivity of tests to detect SARS-CoV-2 from upper respiratory specimens might be insufficient. Repeated rRT-PCR testing of nasopharyngeal swabs was negative for SARS-CoV-2 before the patient was admitted to the intensive care unit. To date, diagnosis of COVID-19 is made mainly on the basis of nucleic acid detection from nasopharyngeal swabs. For suspected cases, 2 negative findings from nasopharyngeal swabs performed $\geq 24$ hours apart would exclude a COVID-19 diagnosis (4). In this case, without the clinicians' persistence because of the patient's travel history, a COVID-19 diagnosis might never have been established. SARS-CoV-2 finally was identified by using mNGS and rRT-PCR of a BALF sample. Therefore, suitable sputum or BALF specimens are necessary to maximize detection in cases of high clinical suspicion; mNGS also might be a helpful tool for identifying SARS-CoV-2 $(1,5)$.

Second, differentiating other causes of respiratory illness from COVID-19 is difficult, especially during influenza season, because common clinical manifestations of COVID-19, including fever, cough, and dyspnea, mimic those of influenza (6-8). In patients with COVID-19, blood tests typically show leucopenia and lymphopenia and most chest computed tomography scans show ground-glass opacity and consolidation with bilateral lung involvement (7-9). Unfortunately, influenza $A$ and other respiratory viruses share these characteristics (10). Co-detection of SARS-CoV-2 and influenza A virus in this case demonstrates that additional challenges to detection remain, especially when patients test negative for SARS-CoV-2 but positive for another virus.

In summary, our case suggests that COVID-19 might be underdiagnosed because of false-negative tests for upper respiratory specimens or co-infection with other respiratory viruses. Broader viral testing might be needed when an apparent etiology is identified, particularly if it would affect clinical management decisions.

This study was supported by the National Key Research and Development Program of China (grant no.

2016YFC1304300 to Q.Z. and grant nos. 2018YFC1200100 and 2018YFC1200102 to B.L.), National Natural Science Foundation of China (grant no. 81870072 to Q.Z.), and Chinese Academy of Medical Sciences Innovation Fund for Medical Sciences (grant no. 2018-I2M-1-003 to Q.Z.).

\section{About the author}

Dr. Wu is a pulmonary and critical care physician specializing in respiratory infection at China-Japan Friendship Hospital, Beijing, China. Her research interests include severe lower respiratory infection and new respiratory infectious diseases.

\section{References}

1. Zhu N, Zhang D, Wang W, Li X, Yang B, Song J, et al.; China Novel Coronavirus Investigating and Research Team. A Novel coronavirus from patients with pneumonia in China, 2019. N Engl J Med. 2020;382:727-33. https:/ / doi.org/ 10.1056/NEJMoa2001017

2. World Health Organization. Novel coronavirus-China. Disease outbreak news: update 12 January [cited 2020 Feb 12]. https:/ / www.who.int/csr/don/12-january-2020-novelcoronavirus-china/en

3. Tan WZX, Zhao X, Ma X, Wang W, Niu P, Xu W, et al. A novel coronavirus genome identified in a cluster of pneumonia cases - Wuhan, China 2019-2020. China CDC Weekly 2020;2:61-62.

4. National Health Commission of People's Republic of China. Diagnosis and treatment for the novel coronavirus pneumonia (trial version 5) [cited 2020 Feb 8]. http://www.nhc.gov.cn/xcs/zhengcwj/202002/ 3b09b894ac9b4204a79db5b8912d4440/files/7260301a393845f c87fcf6dd52965ecb.pdf

5. World Health Organization. Pneumonia of unknown cause-China. Disease outbreak news: 5 January 2020 [cited 2020 Feb 12]. https:/ / www.who.int/csr/don/05-january2020-pneumonia-of-unkown-cause-china

6. Chen N, Zhou M, Dong X, Qu J, Gong F, Han Y, et al. Epidemiological and clinical characteristics of 99 cases of 2019 novel coronavirus pneumonia in Wuhan, China: a descriptive study. Lancet. 2020;395:507-13. https://doi. org/10.1016/S0140-6736(20)30211-7

7. Huang C, Wang Y, Li X, Ren L, Zhao J, Hu Y, et al. Clinical features of patients infected with 2019 novel coronavirus in 
Wuhan, China. Lancet. 2020;395:497-506. https://doi.org/ 10.1016/S0140-6736(20)30183-5

8. Wang D, Hu B, Hu C, Zhu F, Liu X, Zhang J, et al. Clinical characteristics of 138 hospitalized patients with 2019 novel coronavirus-infected pneumonia in Wuhan, China. JAMA. 2020 Feb 7 [Epub ahead of print]. https:/ / doi.org/10.1001/ jama.2020.1585

9. Chan JF, Yuan S, Kok KH, To KK, Chu H, Yang J, et al. A familial cluster of pneumonia associated with the 2019 novel coronavirus indicating person-to-person transmission: a study of a family cluster. Lancet. 2020;395:514-23. https:/ /doi.org/10.1016/S0140-6736(20)30154-9

10. Sullivan SJ, Jacobson RM, Dowdle WR, Poland GA. 2009 H1N1 influenza. Mayo Clin Proc. 2010;85:64-76. https://doi.org/10.4065/mcp.2009.0588

Address for correspondence: Binghuai Lu and Qingyuan Zhan, No. 2, East Yinghua Rd, Chaoyang District, Beijing 1000293, China; email: zs25041@126.com or zhanqycjfh@163.com

\section{Incursions of Candida auris into Australia, 2018}

\author{
Courtney R. Lane, Torsten Seemann, \\ Leon J. Worth, Marion Easton, William Pitchers, \\ Jenny Wong, Donna Cameron, Francesca Azzato, \\ Richard Bartolo, Cristina Mateevici, \\ Caroline Marshall, Monica A. Slavin, \\ Benjamin P. Howden, Deborah A. Williamson
}

Author affiliations: The University of Melbourne at the Peter Doherty Institute for Infection and Immunity, Melbourne, Victoria, Australia (C.R. Lane, T. Seemann, W. Pitchers, D. Cameron, B.P. Howden, D.A. Williamson); VICNISS Coordinating Centre at the Peter Doherty Institute for Infection and Immunity, Melbourne (L.J. Worth); University of Melbourne, Parkville, Victoria, Australia (L.J. Worth, C. Marshall, M.A. Slavin); Peter MacCallum Cancer Centre, Melbourne (L.J. Worth, M.A. Slavin); Victorian Department of Health and Human Services, Melbourne (M. Easton, D. Cameron); Dorevitch Pathology -Western Health, Footscray, Victoria (J. Wong); Royal Melbourne Hospital at the Peter Doherty Institute for Infection and Immunity, Melbourne (F. Azzato, C. Marshall, M.A. Slavin); Western Health, Footscray (R. Bartolo, C. Mateevici); Royal Melbourne Hospital, Parkville (C. Marshall, D.A. Williamson); Austin Health, Heidelberg, Victoria, Australia (B.P. Howden)

DOI: https://doi.org/10.3201/eid2606.190936
Candida auris is an emerging global healthcare-associated pathogen. During July-December 2018, four patients with $C$. auris were identified in Victoria, Australia, all with previous overseas hospitalization. Phylogenetic analysis revealed putative transmission between 2 patients and suspected overseas acquisition in the others. Vigilant screening of at-risk patients is required.

$\mathrm{T}$ he fungal pathogen Candida auris is an emerging global health threat associated with a range of invasive infections, most commonly candidemia; it is often resistant to multiple antifungal drugs (1). First identified in Japan in 2009, C. auris has been reported across all 6 populated continents with outbreaks in healthcare settings, particularly intensive care and high-dependence units $(1,2)$. Four genetic lineages of $C$. auris with phylogeographic variation have been identified (3).

Before July 2018, only 1 case of $C$. auris had been reported in Australia, none in the state of Victoria (population 6.5 million) (4); no centralized surveillance or mandatory reporting has been implemented on a state or national level, and local screening policies are limited. However, Victoria has experienced large interfacility and intrafacility healthcare-associated outbreaks of other multidrug-resistant organisms (5) and has increasingly implemented genomics in both the investigation of outbreaks and routine surveillance (C.R. Lane et al., unpub. data, https:// papers. ssrn.com/sol3/papers.cfm?abstract_id=3498431).

In July 2018, C. auris was cultured from a patient hospitalized in a Victoria healthcare facility. In response, the Victoria Department of Health and Human Services (DHHS) convened an incident management team and issued a Chief Health Officer alert to all health services and laboratories recommending admission screening for patients with recent overseas hospitalization. Also recommended was consideration of $C$. auris in patients with cultured non-C. albicans species and risk factors for fungal infection, including diabetes mellitus and recent antimicrobial drug use. The alert specified that all C. auris and nonspeciated non-C. albicans isolates from high-risk patients be referred to Victorian Public Health laboratories for speciation and characterization and reported to the DHHS (6). We report on the use of genomics to investigate putative transmission of $C$. auris in Victoria during July 1-December 31, 2018.

Isolates of $C$. auris were referred to the Victorian Infectious Diseases Reference Laboratory, where they underwent species identification and antimicrobial susceptibility testing. All isolates were then referred to the Microbiological Diagnostic Unit Public Health Laboratory for DNA extraction, whole-genome sequencing, and bioinformatic analysis. 